Europhysics Letters

PREPRINT

\title{
Resonant tunneling in a Luttinger liquid for arbitrary bar- rier transmission
}

\author{
S. HüGLE and R. EGGER \\ Institut für Theoretische Physik, Heinrich-Heine-Universität, D-40225 Düsseldorf \\ PACS. 71.10.Pm - Fermions in reduced dimensions. \\ PACS. 73.23. Hk - Coulomb blockade, single electron tunneling. \\ PACS. 73.40.Gk - Tunneling.
}

\begin{abstract}
A numerically exact dynamical quantum Monte Carlo approach has been developed and applied to transport through a double barrier in a Luttinger liquid with arbitrary transmission. For strong transmission, we find broad Fabry-Perot Coulomb blockade peaks, with a lineshape parametrized by a single parameter, but at sufficiently low temperatures, nonLorentzian universal lineshapes characteristic of coherent resonant tunneling emerge, even for strong interactions. For weak transmission, our data supports the recently proposed correlated sequential tunneling picture and is consistent with experimental results on intrinsic nanotube dots.
\end{abstract}

Resonant tunneling in a (non-chiral) Luttinger liquid (LL) was studied more than a decade ago [1-3], but has recently attracted widespread attention by theorists again [4-10]. This interest is primarily caused by new experimental realizations of double-barrier setups in interacting 1D quantum wires presumably described by LL theory, using semiconductor quantum wires [11] or nanotubes [12]. The experiments of Ref. [12] have been interpreted in terms of a "correlated sequential tunneling" (CST) mechanism [7,12], since the standard picture of uncorrelated sequential tunneling (UST) in a LL [4] is inconsistent with the observed temperature dependence of the conductance peak height. However, as CST theory essentially relies on a master equation [13], it is clearly of interest to check it against exact results. In this context also other nanotube experiments are of interest, where for nearly transparent doublebarrier, Fabry-Perot oscillations in the gate-voltage dependence of the conductance have been reported [14-17].

As the double-impurity problem in a LL is not integrable, exact solutions covering a wide parameter range are out of reach, and analytical progress has to rely on approximations. One line of reasoning considered very weak Coulomb interactions, i.e. Luttinger parameter $g$ very close to one $[8,9]$, where no CST processes were found. Furusaki has studied the UST regime, where the linewidth of the resonance peak has a linear temperature dependence, and the peak conductance $G_{p} \propto T^{-2+1 / g}$ [4]. The CST mechanism could provide an additional transport channel that may dominate on resonance for strong interactions, and gives instead the observed [12] behavior, $G_{p} \propto T^{-3+2 / g}$ [7]. The CST mechanism is similar but different from conventional cotunneling, which can be the dominant transport channel away from the

(C) EDP Sciences 
resonance. We mention in passing that the exact $g=1 / 2$ solution of a modified model in Ref. [10] neither contains the UST nor the CST regime and therefore cannot resolve this debate. Finally, at low temperatures, instead of sequential tunneling, coherent resonant tunneling is possible, characterized by non-Lorentzian universal lineshapes [1],

$$
G\left(N_{0}, T\right) / G_{0}=f_{g}(X), \quad X=c\left|N_{0}-1 / 2\right| / T^{1-g},
$$

with dimensionful constant $c$ and $G_{0}=e^{2} / h$. Here $N_{0}$ is the dot's average charge induced by external gates, with peak center, say, at $N_{0}=1 / 2$. Obviously, the linewidth then scales as $T^{1-g}$. The universal scaling function has the limiting behavior [1]

$$
1-f_{g}(X \ll 1) \propto X^{2}, \quad f_{g}(X \gg 1) \propto X^{-2 / g} .
$$

While for strong barriers, coherent resonant tunneling is only expected for $g>1 / 2$ [4], for weak barriers (strong transmission), it is largely unclear to what extent this concept applies.

Here we present numerically exact results obtained from a dynamical quantum Monte Carlo approach that provide detailed insight into the strong transmission regime and also sheds light on the controversy about mechanisms of resonant tunneling. This method was successfully used for the corresponding single-barrier case $[18,19]$, and is generalized here to resonant tunneling. We focus on the linear conductance for spinless electrons and symmetric barriers, since additional simulations show that neither spin/flavor degeneracy nor weak asymmetry have a qualitative effect on our results.

We consider a LL containing two impurities of strength $V_{0}$ at $x= \pm d / 2$, thereby forming a quantum dot with LL leads. The single-particle level spacing is $E_{s}=\pi v_{F} / d$, the charging energy is $E_{c}=E_{s} / g^{2}$. In terms of the standard boson field $\phi(x)$ and its conjugate momentum $\Pi(x)$, the Hamiltonian is [1]

$$
H(t)=\frac{v_{F}}{2} \int d x\left\{\Pi^{2}+\frac{1}{g^{2}}\left(\partial_{x} \phi\right)^{2}\right\}+V_{0} \sum_{p= \pm} \cos \left[\sqrt{4 \pi} \phi(p d / 2, t)+e V t+p \pi N_{0}\right],
$$

where $v_{F}$ is the Fermi velocity, $V$ the applied bias voltage, and the current through the dot is

$$
I=G_{0} V+\frac{e}{\sqrt{\pi}}\left\langle\partial_{t} \phi(x, t)\right\rangle
$$

where $x$ is arbitrary and $t \rightarrow \infty$. For $g=1$, refermionization yields the exact conductance $G=d I / d V$. With bandwidth $D$, the dimensionless linewidth

$$
w=\frac{\left(4-\lambda^{2}\right)^{2}}{8 \lambda\left(4+\lambda^{2}\right)}, \quad \lambda=\pi V_{0} / D
$$

and the derivative of the Fermi function, $-d f / d E=1 /\left[4 T \cosh ^{2}(E / 2 T)\right]$, we obtain

$$
\frac{G\left(N_{0}, T\right)}{G_{0}}=\int_{-\infty}^{\infty} d E \frac{-d f}{d E} \frac{w^{2}}{\cos ^{2}\left(\pi\left[N_{0}+E / E_{s}\right]\right)+w^{2}}
$$

where $\hbar=k_{B}=1$. For strong barriers, this leads to the usual Breit-Wigner lineshape with linewidth $w E_{s} / \pi$. Note that the infinite-barrier limit is reached already for $\lambda=2$, where the associated phase shift is in the unitary limit. Equation (6) holds for arbitrary barrier height $V_{0}$, including strong transmission $\left(V_{0} \rightarrow 0\right)$, and allows to firmly establish the validity of our numerical scheme. 
Next we outline our path-integral Monte Carlo (PIMC) approach to the linear conductance for arbitrary $g$. While PIMC is conventionally used to evaluate imaginary-time path integrals, conductance calculations need dynamical information. We first tried to use various schemes to analytically continue imaginary-time PIMC data, but the results were not reliable. This reflects a well-known difficulty related to the numerically ill-posed nature of the analytic continuation [19]. We therefore proceed directly within a (Keldysh) real-time formalism. Although real-time PIMC has to deal with the sign problem, our formulation avoids much of it by mapping the problem to an equivalent Coulomb gas description. In this representation, the sign problem is rather weak and permits numerically exact simulations for the full parameter regime of interest.

Consider the discretized Keldysh contour running from time $t=0$ to $t_{\max }$ and back to zero. We keep $t_{\max }$ finite and define a discrete time spacing $\Delta=t_{\max } / P$ with Trotter number $P$. At time $t_{j}=(j-1) \Delta$, fields $\phi_{j}(x)\left(\phi_{j}^{\prime}(x)\right)$ live on the forward (backward) branch. Next we switch to a Coulomb gas picture by expanding the impurity propagator for sufficiently small $\Delta V_{0}$ [19]. Following Ref. [18], we use a Coulomb gas expansion valid up to order $\left(\Delta V_{0}\right)^{2}$. Introducing "quantum" charges $\xi_{j p}=0, \pm 1, \pm 2$ and "quasiclassical" charges $\eta_{j p}=0, \pm 1 / 2, \pm 1$ for each time $(j=1, \ldots, P)$ and impurity index $(p= \pm)$, where $\eta \pm \xi / 2$ must be integer, it is sufficient to keep $|\eta+\xi / 2|+|\eta-\xi / 2| \leq 2$ within this order of accuracy. Only configurations subject to electroneutrality, $\sum_{j p} \xi_{j p}=0$, contribute to the partition function. Moreover, it turns out that the quasiclassical $\eta$ charges can be summed over analytically $[18,19]$. With $z$ defined in Eq. (7) below, this leads to effective Greens functions $K(\xi, z)$, with the entries $K(0, z)=1-2\left(\Delta V_{0} / 2\right)^{2}(1-\cos z), K( \pm 1, z)= \pm \Delta V_{0} \sin (z / 2)$, and $K( \pm 2, z)=\left(\Delta V_{0} / 2\right)^{2}(1-\cos z)$. Under the Coulomb gas expansion, we can now integrate out all boson fields $\phi_{i}(x)$ and $\phi_{i}^{\prime}(x)$, since they appear only quadratically in the action. Thereby we arrive at an effective action governing the dynamics of the Coulomb gas charges $\{\xi\}$. The result can be put into the language of dissipative quantum mechanics [20] by defining spectral densities $J_{ \pm}(\omega)=\pi g \omega\left[1 \pm \cos \left(\pi \omega / E_{c}\right)\right] e^{-\omega / D}$, with associated correlation function

$$
L_{ \pm}(t)=\int_{0}^{\infty} \frac{d \omega}{\pi} \frac{J_{ \pm}(\omega)}{\omega^{2}} \frac{\cosh [\omega(1 / 2 T-i t)]-\cosh [\omega / 2 T]}{\sinh [\omega / 2 T]} .
$$

This implies (i) the action contribution $\Phi^{\prime}[\{\xi\}]=\sum_{p p^{\prime}} \sum_{j>k} \xi_{j p}\left[S_{+, j k}+p p^{\prime} S_{-, j k}\right] \xi_{k p^{\prime}}$, and (ii) the $z$ 's entering $K(\xi, z)$ are given by

$$
z_{k p}[\{\xi\}]=-2 \sum_{j \geq k} \sum_{p^{\prime}} \xi_{j p^{\prime}}\left(R_{+, j k}+p p^{\prime} R_{-, j k}\right),
$$

with $S_{ \pm, j k}+i R_{ \pm, j k}=L_{ \pm}([j-k] \Delta)$; for the diagonal elements, see Ref. [21].

Collecting results, the conductance is obtained as

$$
G\left(N_{0}, T\right) / G_{0}=1-\lim _{t \rightarrow \infty} \partial_{t} I_{B}(t),
$$

where the function $I_{B}(t)$ can be computed from

$$
I_{B}\left(t_{k}\right)=Z^{-1} \sum_{\{\xi\}} I_{k}[\{\xi\}] \cos \left(\pi N_{0} \sum_{j p} p \xi_{j p}\right) \exp \left(-\Phi^{\prime}[\{\xi\}]\right) \prod_{j p} K\left(\xi_{j p}, z_{j p}\right) .
$$

The normalization $Z$ is the path sum for $I_{k} \rightarrow 1$, and

$$
I_{k}[\{\xi\}]=\Delta \sum_{j^{\prime} p^{\prime}} j^{\prime} \xi_{j^{\prime} p^{\prime}}\left\{\sum_{j p} S_{+, j k} \xi_{j p}+2 \sum_{j \leq k, p} R_{+, k j} \frac{\partial_{z} K\left(\xi_{j p}, z_{j p}\right)}{K\left(\xi_{j p}, z_{j p}\right)}\right\} .
$$




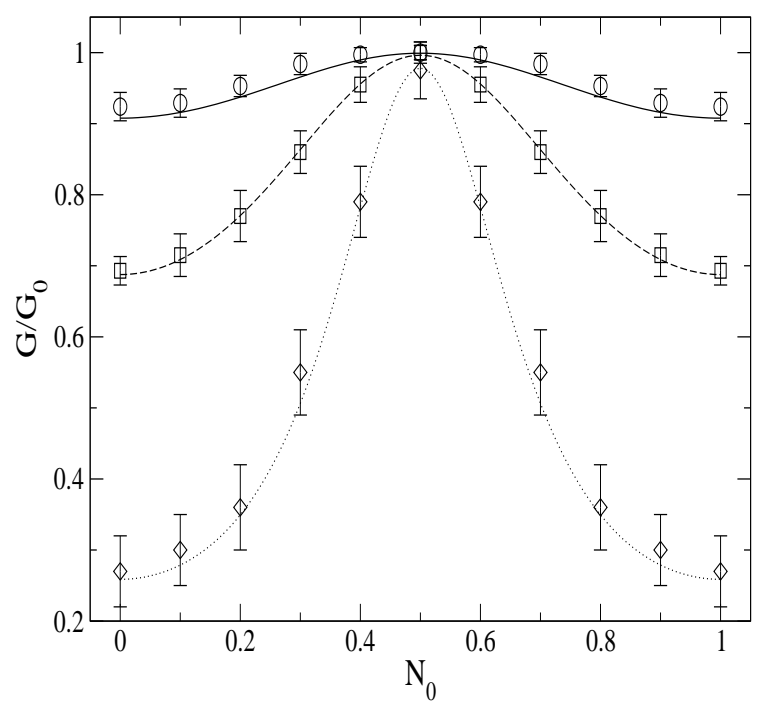

Fig. 1 - Linear conductance versus $N_{0}$ for $g=1, E_{s} / D=\pi / 2, T / D=0.025$, with $V_{0} / D=0.05$ (PIMC: circles; Eq. (6): solid curve), $V_{0} / D=0.1$ (squares; dashed curve), and $V_{0} / D=0.2$ (diamonds; dotted curve).

This is a real-valued quantity, as are all the other quantities appearing in Eq. (9). Remarkably, although we are dealing with a real-time sign problem, it effectively looks just like a fermion one. The sign problem arises because the integrand in Eq. (9) can be negative, leading to interference effects and small signal-to-noise ratio at sufficiently long real times $t_{\max }$.

Monte Carlo trajectories for the quantum fluctuations $\{\xi\}$ are then generated by taking the absolute value of the integrand in Eq. (9) as weight. The average sign in the data reported here was always larger than $\approx 0.001$, which still allows to run stable simulations, albeit sometimes at the expense of very long CPU times. One can obtain the whole function $I_{B}(t)$ in one MC run. For sufficiently long times, this function has a well-defined linear slope which determines the conductance via Eq. (8). Typically, Trotter convergence was reached for discretizations $\Delta V_{0} \leq 0.1$. On a $2 \mathrm{GHz}$ Xeon processor our code performs at an average speed of about $10^{5}$ samples per hour (for $P=40$ ). Several $10^{6} \mathrm{MC}$ samples have to be accumulated to obtain $I_{B}(t)$ for a given parameter set in order to ensure good statistics. Error bars then refer to both standard stochastic $\mathrm{MC}$ errors and to uncertainties from fitting the long-time behavior by a linear slope. The validity and accuracy of our scheme has been established by checking numerical data against the exact $g=1$ solution, Eq. (6), see Fig. 10 The comparison highlights the power of our approach. We then move on to interacting electrons, focusing on $g=0.3$ and $g=0.6$.

Let us start with the case of strong transmission, taking $V_{0} / D=0.05$. Remarkably, for $T / D>0.01$, PIMC data are quantitatively described by the $g=1$ lineshape (6) provided $w=w_{g}(T)$ is treated as a fit parameter. The corresponding values of $w$ are shown in Fig. 2] and reveal power-law behavior, $w_{g}(T) \propto T^{\alpha_{g}}$, with $g$-dependent exponent $\alpha_{g}$. For very weak interactions, a related behavior has been discussed before [9]. Here we find numerical evidence for a power-law temperature dependence of the linewidth for strong interactions. For the two values of $g$ studied, we obtain $\alpha_{0.3} \approx 0.84$ and $\alpha_{0.6} \approx 0.72$. Therefore the strongtransmission peaks become narrower and narrower as $T$ is lowered. Note that each data 


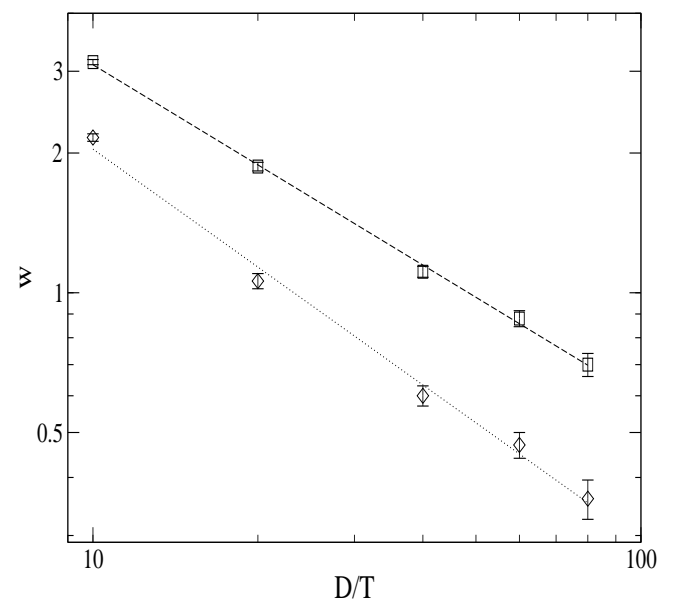

Fig. 2 - Fit parameter $w_{g}(T)$ for conductance lineshape (6) as obtained from QMC data at $g=0.3$ (diamonds) and $g=0.6$ (squares), for $E_{s} / D=\pi / 2$ and $V_{0} / D=0.05$. Dotted and dashed lines are guides to the eye only.

point shown in Fig. 2 actually has been obtained from QMC data for the full conductance lineshape. The lineshape (6) closely resembles experimental results for strong-transmission (Fabry-Perot) oscillations in nanotubes [14-17]. We therefore identify this region of weak barriers and not too low temperature as the Fabry-Perot regime. For $g<1$, such FabryPerot oscillations include remnants of Coulomb blockade effects, which are partly washed out due to pronounced quantum fluctuations present at strong transmission. Nevertheless, these effects are responsible for the narrowing of the resonance peak as temperature is lowered. We mention in passing that no narrow conductance dips were observed such as the ones seen experimentally in Ref. [14]. Such dips are probably related to special impurity scattering processes not contained in the model (3).

At lower temperatures, deviations from the Fabry-Perot lineshape (6) can be seen. However, then our data can be collapsed onto the universal scaling curve (11), see Fig. B] for $g=0.3$. Very similar results were found for $g=0.6$ as well. For small and large $X=c\left|N_{0}-1 / 2\right| / T^{1-g}$, the results in Fig. 3 are consistent with the respective analytical prediction (2). Therefore these universal lineshapes can be identified as coherent resonant tunneling peaks. Although for strong barriers, resonant tunneling exists only for $g>1 / 2$ [1], we observe a perfect resonance peak at $g=0.3$. This is in accordance with renormalization group arguments for weak impurities for $g>1 / 4$ [1], and shows that the picture of coherent resonant tunneling in a Luttinger liquid is actually very robust. Only for very weak barriers and sufficiently high $T$, the Fabry-Perot regime replaces the universal region of resonant tunneling.

Next we discuss stronger barriers, $V_{0} / D=0.2$, where one expects sequential tunneling at

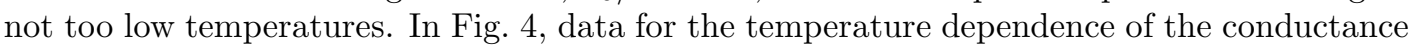
peak height, $G_{p}(T)$, is shown for $g=0.6$. At low temperatures, $G_{p}$ approaches the perfect quantum conductance $G_{0}$ expected in the resonant tunneling regime. With increasing temperature, the conductance goes through a minimum. For intermediate temperatures, but still well below $E_{s}$, sequential tunneling then starts to dominate, and $G_{p}(T)$ increases in a powerlaw fashion, $G_{p}(T) \propto T^{\eta}$. Remarkably, our data are consistent with the CST scaling [7], $\eta=2 / g-3=1 / 3$, but not with the corresponding UST law [4], $\eta=1 / g-2=-1 / 3$, which would even have a different sign. We stress that other exponents, e.g. $\eta=2 / g-2=4 / 3$ as 


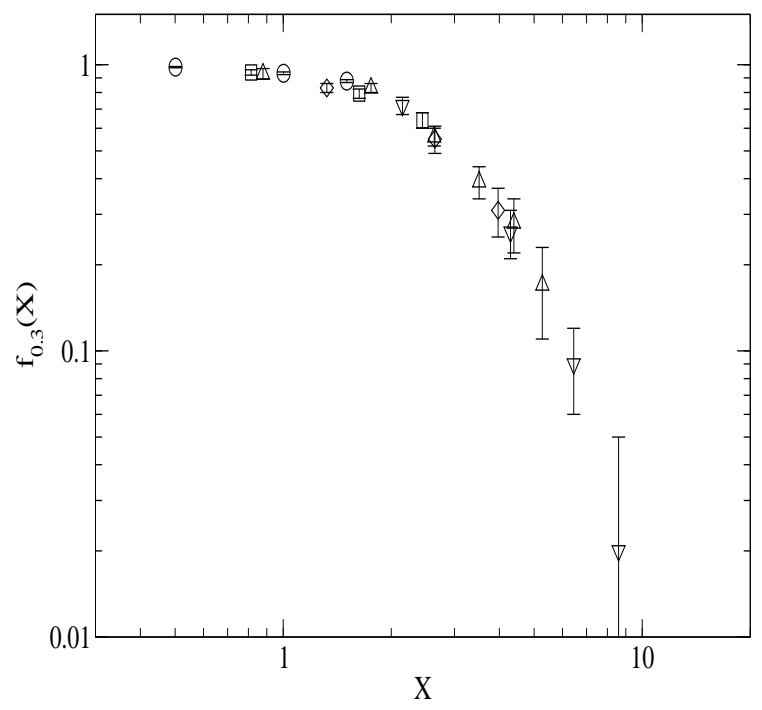

Fig. 3 - Low-temperature QMC data for various $N_{0}, T$ at $E_{s} / D=\pi / 2, V_{0} / D=0.05$, plotted according to Eq. (1) for $g=0.3$. Different symbols refer to different temperatures.

expected for the effective strong single-impurity problem arising at $T \gg E_{s}$ are incompatible with this numerically exact result. Therefore our simulation data suggests that additional mechanisms beyond conventional sequential tunneling can indeed be crucial. As a side remark, we mention that Fig. 4 shows similar high-temperature $\left(T \approx E_{s}\right)$ features as the experimental data of Ref. [12]. The power-law upturn for $G_{p}(T)$ towards the resonant tunneling limit, see

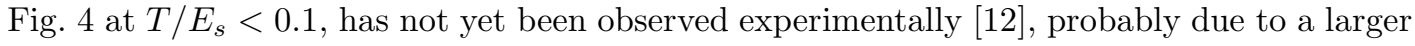

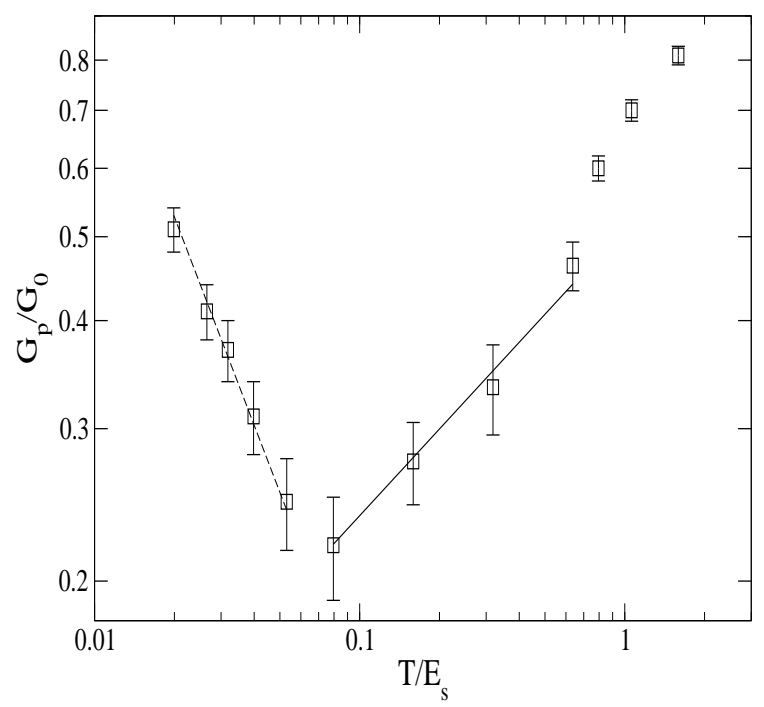

Fig. 4 - Temperature dependence of the peak conductance $G_{p}$ for $g=0.6, V_{0} / D=0.2$, and $E_{s} / D=\pi / 20$. The dashed line reflects a $T^{-0.8}$ power law, the solid line a $T^{1 / 3}$ law. 
barrier height $V_{0}$ in the samples.

To conclude, we have developed and applied a numerically exact and well-controlled realtime Monte Carlo approach to the computation of the resonant tunneling conductance in a Luttinger liquid. For weak barriers and not too low temperature, we identify a Fabry-Perot regime, where the conductance peak has a lineshape given by Eq. (6) with a temperaturedependent linewidth parameter $w_{g}(T)$. Within the range of applicability, $w_{g}(T)$ exhibits $g$-dependent power-law scaling. At sufficiently low temperatures, for $g>1 / 4$, we then find a crossover into the universal coherent resonant tunneling regime. At higher temperatures, we find numerical evidence in support of the recently suggested correlated sequential tunneling picture [7]. Finally, we note that this algorithm also allows to study out-of-equilibrium transport, quantum shot noise, and transport in the presence of a Kondo-type dot.

We thank A.O. Gogolin, M. Grifoni, A. Komnik, V. Meden, K. Schönhammer, and M. Thorwart for useful discussions. This work has been supported by the EU and by the DFG.

\section{REFERENCES}

[1] Kane C. L. and Fisher M. P. A., Phys. Rev. B, 46 (1992) 15233.

[2] Furusaki A. and Nagaosa N., Phys. Rev. B, 37 (1993) 3827.

[3] Moon K., Yi H., Kane C. L., Girvin S. M. and Fisher M. P. A., Phys. Rev. Lett., 71 (1993) 4381.

[4] Furusaki A., Phys. Rev. B, 57 (1998) 7141.

[5] Kleimann T., Cavaliere F., Sassetti M. and Kramer B., Phys. Rev. B, 66 (2002) 165311.

[6] Braggio A., Sassetti M. and Kramer B., Phys. Rev. Lett., 87 (2001) 146802.

[7] Thorwart M., Grifoni M., Cuniberti G., Postma H. W. Ch. and Dekker C., Phys. Rev. Lett., 89 (2002) 196402. The assumption of finite-range interactions made in that paper is overly restrictive and can be released. The CST theory should therefore apply to the standard zero-range LL model.

[8] Nazarov Yu. and Glazman L. I., Phys. Rev. Lett., 91 (2003) 126804.

[9] Polyakov D. G. and Gornyi I. V., Phys. Rev. B, 68 (2003) 035421.

[10] Komnik A. and Gogolin A. O., Phys. Rev. Lett., 90 (2003) 246403.

[11] Auslaender O. M., Yacoby A., de Picciotto R., Baldwin K. W., Pfeiffer L. N. and West K. W., Phys. Rev. Lett., 84 (2000) 1764.

[12] Postma H.W.Ch., Teepen T., Yao Z., Grifoni M. and Dekker C., Science, 76 (2001) 293.

[13] Thorwart M., Grifoni M. and Egger R., unpublished.

[14] Liang W., Bockrath M., Bozovic D., Hafner J. H., Tinkham M. and Park H., Nature, 411 (2001) 665.

[15] Kong J., Yenilmez E., Tombler T. W., Kim W., Dai H., Laughlin R.B., Liu L., Jayanthi C. S. and Wu S., Phys. Rev. Lett., 87 (2001) 106801.

[16] Bockrath M., Liang W., Bozovic D., Hafner J. H., Lieber C. M., Tinkham M. and PARK H., Science, 291 (2001) 283.

[17] Park J. W., Choi J. B. and Yoo K.-H., Appl. Phys. Lett., 81 (2002) 2644.

[18] Mak C. H. and Egger R., Phys. Rev. E, 49 (1994) 1997.

[19] Leung K., Egger R. and Mak C. H., Phys. Rev. Lett., 75 (1995) 3344.

[20] Sassetti M., Napoli F. and Weiss U., Phys. Rev. B, 52 (1995) 11213.

[21] Egger R. and Mak C. H., J. Phys. Chem., 98 (1994) 9903. 